\title{
Study of heat transport by stationary magneto-convection in a Newtonian liquid under temperature or gravity modulation using Ginzburg-Landau model
}

\author{
P.G. Siddheshwar ${ }^{a}$, B.S. Bhadauria ${ }^{\text {b,c,*, }}$ Pankaj Mishra ${ }^{c}$, Atul K. Srivastava ${ }^{c}$ \\ a Department of Mathematics, Bangalore University, Bangalore 560001, India \\ b Department of Applied Mathematics and Statistics, School for Physical Sciences, Babasaheb Bhimrao Ambedkar University, Lucknow 226025, India

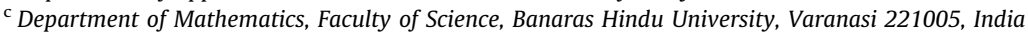

\section{A R T I C L E I N F O}

\section{Article history:}

Received 29 May 2011

Received in revised form

23 June 2011

Accepted 28 June 2011

Available online 3 September 2011

Keywords:

Magneto-convection

Temperature modulation

Gravity modulation

Rayleigh number

Newtonian liquid

\begin{abstract}
A B S T R A C T
The present paper deals with a weak non-linear stability problem of magneto-convection in an electrically conducting Newtonian fluid, confined between two horizontal surfaces, under a constant vertical magnetic field, and subjected to an imposed time-periodic boundary temperature (ITBT) or gravity modulation (ITGM). In the case of ITBT, the temperature gradient between the walls of the fluid layer consists of a steady part and a time-dependent oscillatory part. The temperature of both walls is modulated in this case. In the problem involving ITGM, the gravity field has two parts: a constant part and an externally imposed time periodic part, which can be realized by oscillating the fluid layer. The disturbance is expanded in terms of power series of amplitude of convection, which is assumed to be small. Using Ginzburg-Landau equation, the effect of modulations on heat transport is analyzed. Effect of various parameters on the heat transport is also discussed.
\end{abstract}

(c) 2011 Published by Elsevier Ltd.

\section{Introduction}

There are many interesting situations of practical importance in which the temperature gradient is a function of both space and time. This uniform temperature gradient (temperature modulation) can be determined by solving the energy equation with suitable time-dependent thermal boundary conditions and can be used as an effective mechanism to control the convective flow. Predictions exist for a variety of responses to modulation depending on the relative strength and rate of forcing. Among these, there are the upward or downward shift of convective threshold compared to the unmodulated problems. Lot of work is available in the literature covering how a time-periodic boundary temperature affects the onset of Rayleigh-Bénard convection. An excellent review related to this problem is given by [1].

The effect of temperature modulation on thermal stability in a viscous fluid layer was first considered by Venezian [2]. Performing a linear stability analysis of small amplitude temperature modulation, he derived the onset criteria using a perturbation expansion in powers of the amplitude of oscillation. He showed that the onset of

\footnotetext{
* Corresponding author at: Department of Mathematics, Faculty of Science, Banaras Hindu University, Varanasi 221005, India.

E-mail addresses: pgsiddheshwar@hotmail.com (P.G. Siddheshwar), mathsbsb@yahoo.com,drbsbhadauria@yahoo.com (B.S. Bhadauria), pankaj1mishra@gmail.com (P. Mishra), atulshaswat@gmail.com (A.K. Srivastava).
}

convection can be delayed or advanced depending on the values of the parameters as well as on the types of modulation. Using Galerkin technique and Floquet theory, Rosenblat and Tanaka [3] studied the effect of thermal modulation on the onset of RayleighBénard convection for rigid-rigid boundaries. The first non-linear problem of thermal instability under temperature modulation was studied by Roppo et al. [4]. They observed that ranges of stable hexagons are produced by the modulation effect near the critical Rayleigh number. Bhadauria and Bhatia [5] studied the effect of temperature modulation on thermal instability by considering rigid-rigid boundaries and different types of temperature profiles. Also Bhadauria [6] studied the effect of temperature modulation respectively, under vertical magnetic field. Further Bhadauria et al. [7] studied the non-linear aspects of thermal instability under temperature modulation, considering various temperature profiles.

The problem of convection in a fluid layer in the presence of complex body forces has gained considerable attention in recent decades due to its promising applications in engineering and technology. The time-dependent gravitational field, one of the complex forces, is of interest in space laboratory experiments, in areas of crystal growth and others. It is also of importance in the large-scale convection in atmosphere. The random fluctuations of gravity field, both in magnitude and direction, experienced in space laboratories, significantly influence natural convection. Existence of adverse density variations within the fluid and a body force are the necessary conditions to initiate natural convection. The idea of using 


\begin{tabular}{|c|c|c|c|}
\hline \multicolumn{2}{|c|}{ Nomenclature } & $\rho$ & fluid density \\
\hline \multirow{2}{*}{\multicolumn{2}{|c|}{ Latin Symbols }} & $\omega$ & Modulation frequency \\
\hline & & $\delta_{1}$ & amplitude of gravity modulation \\
\hline$A$ & amplitude of streamline perturbation & $\begin{array}{l}\delta_{2} \\
\phi\end{array}$ & $\begin{array}{l}\text { amplitude of temperature modulation } \\
\text { phase angle }\end{array}$ \\
\hline$d$ & height of the fluid layer & $\varepsilon$ & perturbation parameter \\
\hline$g$ & acceleration due to gravity & $\Psi$ & stream function \\
\hline$k_{c}$ & wave number & $\Psi^{*}$ & dimensionless stream function \\
\hline$\delta^{2}$ & $k_{c}^{2}+\pi^{2}$ & $\Theta^{\prime}$ & temperature perturbation \\
\hline $\mathrm{Nu}$ & Nusselt number & $\Theta^{*}$ & dimensionless temperature perturbation \\
\hline$p$ & pressure & $\Phi$ & magnetic potential \\
\hline $\mathrm{Pm}$ & magnetic Prandtl number $\left(v_{m} / \kappa_{T}\right)$ & $\Phi^{*}$ & dimensionless magnetic potential \\
\hline $\operatorname{Pr}$ & Prandtl number $\left(v / \kappa_{T}\right)$ & $s$ & $s=\varepsilon^{2} t($ small time scale $)$ \\
\hline$Q$ & Chandarsekhar number $\left(\mu_{m} H_{b}^{2} d^{2} / \rho_{0} v v_{m}\right)$ & $J$ & \\
\hline & $\begin{array}{l}\text { Rayleigh number }\left(\alpha g \Delta T d^{3} / \nu \kappa_{T}\right) \\
\text { critical Rayleigh number }\left(\delta^{2}\left(\delta^{4}+Q \pi^{2}\right) / k_{c}^{2}\right)\end{array}$ & \multicolumn{2}{|c|}{ Other symbols } \\
\hline$T$ & temperature & \multirow{2}{*}{$\nabla^{2}$} & $\partial^{2} \quad \partial^{2}$ \\
\hline$\Delta T$ & temperature difference across the fluid layer & & $\overline{\partial x^{2}}+\overline{\partial z^{2}}$ \\
\hline & time & \multirow{2}{*}{\multicolumn{2}{|c|}{ Subscripts }} \\
\hline$x, y, z$ & space co-ordinates & & \\
\hline \multirow{2}{*}{\multicolumn{2}{|c|}{ Greek symbols }} & $b$ & Basic state \\
\hline & & $0 c$ & Critical value \\
\hline$\alpha$ & coefficient of thermal expansion & 0 & Value of the un-modulated case \\
\hline$\mu_{m}$ & magnetic permeability & \multirow{2}{*}{\multicolumn{2}{|c|}{ Superscripts }} \\
\hline$v_{m}$ & magnetic viscosity & & \\
\hline$\kappa_{T}$ & thermal diffusivity & & \\
\hline$\mu$ & dynamic coefficient of viscosity of the fluid & ' & perturbed quantity \\
\hline$v$ & kinematic viscosity $\left(\mu / \rho_{0}\right)$ & $*$ & dimensionless quantity \\
\hline
\end{tabular}

mechanical vibration as a tool to improve the heat transfer rate has received much attention.

Gresho and Sani [8] and Greshuni and Zhukhovitskii [9] were the first to study the effect of gravity modulation in a fluid layer. Biringen and Peltier [10] investigated, numerically, the non-linear three dimensional Rayleigh-Bénard problem under gravity modulation, and confirmed the result of Gresho and Sani [8]. Clever et al. [11] performed a detailed non-linear analysis of the problem and presented the stability limits to a much wider region of parameter space. Shu et al. [12] examined the effects of modulation of gravity and thermal gradients on natural convection in a cavity, numerically as well as experimentally. They found that for low Prandtl number fluids, modulations in gravity and temperature produce the same flow field both in structure and in magnitude. Clever et al. [13], Rogers et al. [14], Aniss et al. $[15,16]$, and Bhadauria et al. [17] showed that the gravitational modulation, which can be realized by vertically oscillating a horizontal liquid layer, acts on the entire volume of liquid and may have a stabilizing or destabilizing effect depending on the amplitude and frequency of the forcing. Boulal et al. [18] focused attention on the influence of a quasi-periodic gravitational modulation on the convective instability threshold. They predicted that the threshold of convection corresponds precisely to quasiperiodic solutions.

Magneto-convection arises due to the interaction of electrically conducting fluid flow and the applied magnetic field. Ozoe and Maruo [19] investigated magnetic and gravitational natural convection of metal silicon and performed two-dimensional numerical computations to obtain the rate of heat transfer. Siddheshwar and Pranesh [20] examined the effects of time-periodic temperature/gravity modulation on the onset of magneto-convection in electrically conducting fluids with internal angular momentum by making a linear stability analysis.
In this paper, we use the Ginzburg-Landau (GL) equation to examine non-linear magneto-convection in an electrically conducting Newtonian liquid. GL equations arise as a solvability condition in a wide variety of problems in continuum mechanics while dealing with a weakly non-linear stability of systems.

To the best of authors' knowledge no study is available in which non-linear study of the effect of temperature modulation or gravity modulation on magneto-convection in a Newtonian liquid is considered using Ginzburg-Landau equation. Therefore, in this paper, we study non-linear magneto-convection in the presence of ITBT or ITGM.

\section{Mathematical formulation}

We consider an electrically conducting liquid of depth $d$, confined between two infinite, parallel, horizontal planes at $z=0$ and $z=d$. Cartesian co-ordinates have been taken with the origin at the bottom of the liquid layer, and the $z$-axis vertically upwards. The surfaces are maintained at a constant gradient $\Delta T / d$ and a constant magnetic field $H_{b} \hat{k}$ is applied across the liquid region (as shown in Fig. 1). Under the Boussinesq approximation, the dimensional governing equations for the study of magnetoconvection in an electrically conducting liquid are

$$
\begin{aligned}
& \frac{\partial \vec{q}}{\partial t}+(\vec{q} \cdot \nabla) \vec{q}=-\frac{1}{\rho_{0}} \nabla p+\frac{\rho}{\rho_{0}} \vec{g}+\frac{\mu}{\rho_{0}} \nabla^{2} \vec{q}+\frac{\mu_{m}}{\rho_{0}} \vec{H} \cdot \nabla \vec{H}, \\
& \frac{\partial T}{\partial t}+(\vec{q} \cdot \nabla) T=\kappa_{T} \nabla^{2} T, \\
& \frac{\partial \vec{H}}{\partial t}+(\vec{q} \cdot \nabla) \vec{H}-(\vec{H} \cdot \nabla) \vec{q}=v_{m} \nabla^{2} \vec{H},
\end{aligned}
$$




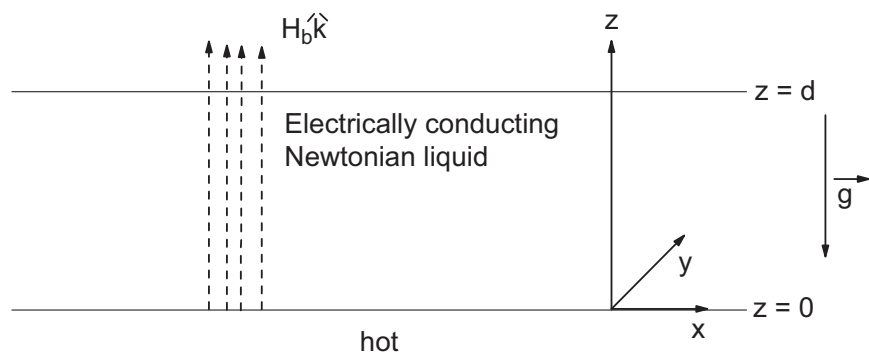

Fig. 1. Schematic of physical configuration.

$\nabla \cdot \vec{q}=0$

$\nabla \cdot \vec{H}=0$.

The constants and variables in the above equations have their usual meanings as given in the nomenclature. The relation between the density and temperature is given by

$\rho=\rho_{0}\left[1-\alpha\left(T-T_{0}\right)\right]$.

In our study we first consider the effect of temperature modulation (time-periodic boundary temperatures) and then the effect of gravity modulation (time-periodic body force) on heat transport by magneto-convection.

For temperature modulation of the boundaries, we consider the imposed surface temperature conditions as

$$
\begin{aligned}
T(t) & =T_{0}+\frac{\Delta T}{2}\left[1+\varepsilon^{2} \delta_{2} \cos \omega t\right] \quad \text { at } z=0 \\
& =T_{0}-\frac{\Delta T}{2}\left[1-\varepsilon^{2} \delta_{2} \cos (\omega t+\phi)\right] \quad \text { at } z=d .
\end{aligned}
$$

Here $\delta_{2}$ represents the amplitude of modulation and $\varepsilon$ (to be defined later in the next section) indicates the smallness of the amplitude, $\Delta T$ is the temperature difference, $\omega$ is the modulation frequency and $\phi$ is the phase angle.

The basic state of fluid is quiescent and is given by

$\vec{q}_{b}=0, \quad p=p_{b}(z, t), \quad T=T_{b}(z, t), \quad \rho=\rho_{b}(z, t), \quad \vec{H}_{b}=H_{b} \hat{k}$,

$\frac{\partial T_{b}}{\partial t}=\kappa_{T} \frac{\partial^{2} T_{b}}{\partial z^{2}}$

$\frac{\partial p_{b}}{\partial z}=-\rho_{b} g$

$\rho_{b}=\rho_{0}\left[1-\alpha\left(T-T_{0}\right)\right]$.

The solution of Eq. (9), subject to the boundary conditions (7), is given by

$T_{b}(z, t)=T_{S}(z)+\varepsilon^{2} \delta_{2} \operatorname{Re}\left[T_{1}(z, t)\right]$,

where

$T_{S}(z)=T_{0}+\frac{\Delta T}{2}\left(1-\frac{2 z}{d}\right)$,

$T_{1}(z, t)=\left[a(\lambda) e^{\lambda z / d}+a(-\lambda) e^{-\lambda z / d}\right] e^{-i \omega t}$,

$\lambda^{2}=-i \omega d^{2} / \kappa_{T} \quad$ and $\quad a(\lambda)=\frac{\Delta T}{2} \frac{e^{-i \phi}-e^{-\lambda}}{e^{\lambda}-e^{-\lambda}}$.

In the above equations $T_{S}(z)$ is the steady temperature field and $T_{1}$ is the oscillating part of $T_{b}$, while Re stands for the real part. The basic pressure field can also be obtained by solving Eq. (10) but it is not required explicitly.
We assume finite amplitude perturbations to the basic state in the form:

$\vec{q}=\vec{q}_{b}+\vec{q}^{\prime}, \quad T=T_{b}+\Theta^{\prime}, \quad p=p_{b}+p^{\prime}, \quad \rho=\rho_{b}+\rho^{\prime}, \quad \vec{H}=\vec{H}_{b}+\vec{H}^{\prime}$.

Substituting Eq. (16) in the Eqs. (1)-(6), we get the following equations:

$\nabla \cdot \overrightarrow{q^{\prime}}=0$

$\frac{\partial \overrightarrow{q^{\prime}}}{\partial t}+\left(\overrightarrow{q^{\prime}} \cdot \nabla\right) \overrightarrow{q^{\prime}}-\frac{\mu_{m}}{\rho_{0}}\left(\overrightarrow{H^{\prime}} \cdot \nabla\right) \overrightarrow{H^{\prime}}=-\frac{1}{\rho_{0}} \nabla p^{\prime}-\frac{\rho^{\prime}}{\rho_{0}} g \hat{k}+v \nabla^{2} \vec{q}^{\prime}$

$+\frac{\mu_{m}}{\rho_{0}} H_{b} \frac{\partial H^{\prime}}{\partial z}$,

$\frac{\partial \Theta^{\prime}}{\partial t}+\left(\overrightarrow{q^{\prime}} \cdot \nabla\right) \Theta^{\prime}+w^{\prime} \frac{\partial T_{b}}{\partial z}=\kappa_{T} \nabla^{2} \Theta^{\prime}$,

$\frac{\partial \vec{H}^{\prime}}{\partial t}+\left(\vec{q}^{\prime} \cdot \nabla\right) \vec{H}^{\prime}-\left(\vec{H}^{\prime} \cdot \nabla\right) \vec{q}^{\prime}-H_{b} \frac{\partial w^{\prime}}{\partial z}=v_{m} \nabla^{2} \vec{H}^{\prime}$,

$\rho^{\prime}=-\rho_{0} \alpha \Theta^{\prime}$

Further we consider only two-dimensional disturbances in our study and hence the stream function $\Psi$ and magnetic potential $\Phi$ are introduced as

$u^{\prime}=\frac{\partial \Psi}{\partial z}, \quad w^{\prime}=-\frac{\partial \Psi}{\partial x}, \quad H_{x}^{\prime}=\frac{\partial \Phi}{\partial z}, \quad H_{z}^{\prime}=-\frac{\partial \Phi}{\partial x}$.

We eliminate density between Eqs. (18) and (21) and then pressure term from the resulting equation by taking curl of it. The resulting system is then non-dimensionalized using the following scales:

$\Psi=\kappa_{T} \Psi^{*}, \quad(x, y, z)=d\left(x^{*}, y^{*}, z^{*}\right), \quad \Theta=\Delta T \Theta^{*}, \quad \Phi=d H_{b} \Phi^{*}, \quad t=t^{*} \frac{d^{2}}{\kappa_{T}}$.

After dropping the asterisk, we get the non-dimensional governing equations in the form:

$$
\begin{gathered}
\frac{1}{\operatorname{Pr}} \frac{\partial\left(\nabla^{2} \Psi\right)}{\partial t}+R \frac{\partial \Theta}{\partial x}-\nabla^{4} \Psi-Q P m \frac{\partial}{\partial z}\left(\nabla^{2} \Phi\right) \\
=\frac{1}{\operatorname{Pr}} \frac{\partial\left(\Psi, \nabla^{2} \Psi\right)}{\partial(x, z)}-Q P m \frac{\partial\left(\Phi, \nabla^{2} \Phi\right)}{\partial(x, z)} \\
\left(\frac{\partial}{\partial t}-\nabla^{2}\right) \Theta-\frac{\partial \Psi}{\partial x} \frac{\partial T_{b}}{\partial z}=\frac{\partial(\Psi, \Theta)}{\partial(x, z)} \\
\left(\frac{\partial}{\partial t}-P m \nabla^{2}\right) \Phi-\frac{\partial \Psi}{\partial z}=\frac{\partial(\Psi, \Phi)}{\partial(x, z)}
\end{gathered}
$$

where, $P m=v_{m} / \kappa_{T}$ is magnetic Prandtl number, $P r=v / \kappa_{T}$ is Prandtl number, $R=\alpha g \Delta T d^{3} / \nu \kappa$ is Rayleigh number and $Q=\mu_{m} H_{b}^{2} d^{2} / \varrho_{0} v v_{m}$ is Chandrasekhar number.

Non-dimensional form of the temperature gradient $\partial T_{b} / \partial z$ which appears in Eq. (24) can be obtained from (12) as

$T_{b}(z, t)=T_{0}+(1-z)+\varepsilon^{2} \delta_{2} F(z, t)$,

where

$F(z, t)=\operatorname{Re}\left[\left\{A(\lambda) e^{\lambda z}+A(-\lambda) e^{-\lambda z}\right\} e^{-i \omega t}\right]$,

$A(\lambda)=\frac{1}{2} \frac{\left(e^{-i \phi}-e^{-\lambda}\right)}{\left(e^{\lambda}-e^{-\lambda}\right)}, \quad \lambda=(1-i) \sqrt{\frac{\omega}{2}}$

Now, to study stationary magneto-convection, we use the time variations only at the slow time scale $s=\varepsilon^{2} t$. Using this, we can 
write the matrix form of Eqs. (23)-(25) as follows:

$$
\begin{aligned}
& {\left[\begin{array}{ccc}
\frac{\varepsilon^{2}}{P_{r}} \frac{\partial}{\partial s} \nabla^{2}-\nabla^{4} & R \frac{\partial}{\partial x} & -Q P m \frac{\partial}{\partial z} \nabla^{2} \\
\left\{1-\varepsilon^{2} \delta_{2} F^{\prime}(z, s)\right\} \frac{\partial}{\partial x} & \varepsilon^{2} \frac{\partial}{\partial s}-\nabla^{2} & 0 \\
-\frac{\partial}{\partial z} & 0 & \varepsilon^{2} \frac{\partial}{\partial s}-P m \nabla^{2}
\end{array}\right]\left[\begin{array}{l}
\Psi \\
\Theta \\
\Phi
\end{array}\right]} \\
& =\left[\begin{array}{c}
\frac{1}{P_{r}} \frac{\partial\left(\Psi, \nabla^{2} \Psi\right)}{\partial(x, z)}-Q P m \frac{\partial\left(\Phi, \nabla^{2} \Phi\right)}{\partial(x, z)} \\
\frac{\partial(\Psi, \Theta)}{\partial(x, z)} \\
\frac{\partial(\Psi, \Phi)}{\partial(x, z)}
\end{array}\right] .
\end{aligned}
$$

The boundary condition to solve these equations are

$\Psi=0=\nabla^{2} \Psi, \quad \Theta=D \Phi=0$ on $\mathrm{z}=0$,

$\Psi=0=\nabla^{2} \Psi, \quad \Theta=D \Phi=0$ on $\mathrm{z}=1$.

where $D=\partial / \partial z$.

\section{Amplitude equation (Ginzburg-Landau equation) and heat transport}

We now introduce the following asymptotic expansions in the above system (28):

$R=R_{0 c}+\varepsilon^{2} R_{2}+\varepsilon^{4} R_{4}+\cdots$,

$\Psi=\varepsilon \Psi_{1}+\varepsilon^{2} \Psi_{2}+\varepsilon^{3} \Psi_{3}+\cdots$,

$\Theta=\varepsilon \Theta_{1}+\varepsilon^{2} \Theta_{2}+\varepsilon^{3} \Theta_{3}+\cdots$,

$\Phi=\varepsilon \Phi_{1}+\varepsilon^{2} \Phi_{2}+\varepsilon^{3} \Phi_{3}+\cdots$

We substitute Eqs. (30)-(33) in Eq. (28), then at the lowest order in $\varepsilon$ the equations are:

$\left[\begin{array}{ccc}-\nabla^{4} & R_{0} \frac{\partial}{\partial x} & -Q P m \frac{\partial}{\partial z} \nabla^{2} \\ \frac{\partial}{\partial x} & -\nabla^{2} & 0 \\ -\frac{\partial}{\partial z} & 0 & -P m \nabla^{2}\end{array}\right]\left[\begin{array}{l}\Psi_{1} \\ \Theta_{1} \\ \Phi_{1}\end{array}\right]=\left[\begin{array}{l}0 \\ 0 \\ 0\end{array}\right]$.

This corresponds to the linear stability equations for stationary magneto-convection and has the solution in the form:

$\Psi_{1}(x, z, s)=A(s) \sin \left(k_{c} x\right) \sin (\pi z)$,

$\Theta_{1}(x, z, s)=-\frac{k_{c}}{\delta^{2}} A(s) \cos \left(k_{c} x\right) \sin (\pi z)$,

$\Phi_{1}(x, z, s)=\frac{\pi}{P m \delta^{2}} A(s) \sin \left(k_{c} x\right) \cos (\pi z)$,

where $\delta^{2}=k_{c}^{2}+\pi^{2}$. Further the critical values of the Rayleigh number for the onset of magneto-convection in the absence of temperature modulation is

$R_{0 c}=\frac{\delta^{2}\left(\delta^{4}+Q \pi^{2}\right)}{k_{c}^{2}}$.

At the second order, we have

$\left[\begin{array}{ccc}-\nabla^{4} & R_{0} \frac{\partial}{\partial x} & -Q P m \frac{\partial}{\partial z} \nabla^{2} \\ \frac{\partial}{\partial x} & -\nabla^{2} & 0 \\ -\frac{\partial}{\partial z} & 0 & -P m \nabla^{2}\end{array}\right]\left[\begin{array}{l}\Psi_{2} \\ \Theta_{2} \\ \Phi_{2}\end{array}\right]=\left[\begin{array}{l}R_{21} \\ R_{22} \\ R_{23}\end{array}\right]$,

where

$R_{21}=\frac{1}{P_{r}} \frac{\partial\left(\Psi_{1}, \nabla^{2} \Psi_{1}\right)}{\partial(x, z)}-Q P m \frac{\partial\left(\Phi_{1}, \nabla^{2} \Phi_{1}\right)}{\partial(x, z)}=0$,

$R_{22}=\frac{\partial\left(\Psi_{1}, \Theta_{1}\right)}{\partial(x, z)}=-\frac{k_{c}^{2} \pi}{2 \delta^{2}} A^{2}(s) \sin (2 \pi z)$ and

$R_{23}=\frac{\partial\left(\Psi_{1}, \Phi_{1}\right)}{\partial(x, z)}=-\frac{k_{c} \pi^{2}}{2 \tau \delta^{2}} A^{2}(s) \sin \left(2 k_{c} x\right)$

Now considering the conditions $\Psi_{2}(x, z, s)=0, \partial \Theta_{2} / \partial x=0$ and $\partial \Phi_{2} / \partial z=0$, the solution of the second order system is

$\Psi_{2}(x, z, s)=0$,

$\Theta_{2}(z, s)=-\frac{k_{c}}{8 \pi \delta^{2}} A^{2}(s) \sin (2 \pi z)$,

$\Phi_{2}(x, s)=-\frac{\pi^{2}}{8 k_{c} P m^{2} \delta^{2}} A^{2}(s) \sin \left(2 k_{c} x\right)$.

The horizontally averaged Nusselt number, $\mathrm{Nu}$, for the stationary magneto-convection (the preferred mode in this problem) is given by

$N u(s)=\frac{\left[\frac{k_{c}}{2 \pi} \int_{0}^{2 \pi / k_{c}}\left(1-z+\Theta_{2}\right) d x\right]_{z=0}}{\left[\frac{k_{c}}{2 \pi} \int_{0}^{2 \pi / k_{c}}(1-z) d x\right]_{z=0}}$.

One must note here that it is only $T_{s}=1-z$ that contributes explicitly to $N u(s)$ due to the fact that $F(z, t)$ is effective at $O\left(\varepsilon^{2}\right)$. Substituting (38b) in (39) and simplifying, we get

$N u(s)=1+\frac{k_{c}^{2}}{4 \delta^{2}}[A(s)]^{2}$.

At the third order, we have

$\left[\begin{array}{ccc}-\nabla^{4} & R_{0} \frac{\partial}{\partial x} & -Q P m \frac{\partial}{\partial z} \nabla^{2} \\ \frac{\partial}{\partial x} & -\nabla^{2} & 0 \\ -\frac{\partial}{\partial z} & 0 & -P m \nabla^{2}\end{array}\right]\left[\begin{array}{l}\Psi_{3} \\ \Theta_{3} \\ \Phi_{3}\end{array}\right]=\left[\begin{array}{l}R_{31} \\ R_{32} \\ R_{33}\end{array}\right]$,

where

$R_{31}=\frac{Q \pi^{4} A^{3}}{P m^{2} \delta^{2}}\left[\frac{1}{4}-\frac{k_{c}^{2}}{\delta^{2}}\right] \sin \left(k_{c} x\right) \cos \left(2 k_{c} x\right) \sin (\pi z)$

$+\frac{\delta^{2}}{P_{r}} \frac{d A}{d s} \sin \left(k_{c} x\right) \sin (\pi z)-R_{2} \frac{k_{c}^{2}}{\delta^{2}} A \sin \left(k_{c} x\right) \sin (\pi z)$,

$R_{32}=-\frac{A^{3} k_{c}^{3}}{4 \delta^{2}} \cos \left(k_{c} x\right) \sin (\pi z) \cos (2 \pi z)$

$+\frac{k_{c}}{\delta^{2}} \frac{d A}{d s} \cos \left(k_{c} x\right) \sin (\pi z)+\delta_{2} k_{c} F(z, s) A \cos \left(k_{c} x\right) \sin (\pi z)$,

$R_{33}=\frac{A^{3} \pi^{3}}{4 \tau^{2} \delta^{2}} \cos (\pi z) \sin \left(k_{c} x\right) \cos \left(2 k_{c} x\right)-\frac{\pi}{P m \delta^{2}} \frac{d A}{d s} \sin \left(k_{c} x\right) \cos (\pi z)$.

We now apply the solvability condition for the existence of the solution of the third order system and simplify to get the GinzburgLandau equation in the form:

$$
\begin{aligned}
{\left[\frac{\delta^{4}}{\operatorname{Pr}}+\frac{R_{0} k_{c}^{2}}{\delta^{4}}-\frac{Q \pi^{2}}{P m \delta^{2}}\right] \frac{d A(s)}{d s}=} & R_{0 c} \frac{k_{c}^{2}}{\delta^{2}}\left[R_{2}^{*}-2 \delta_{2} I\right] A(s) \\
& -\left[\frac{R_{0 c} k_{c}^{4}}{8 \delta^{4}}-\frac{Q \pi^{4}}{4 \tau^{2} \delta^{2}}+\frac{Q \pi^{4} k_{c}^{2}}{2 \tau^{2} \delta^{4}}\right] A^{3}(s),
\end{aligned}
$$

where $I=\int_{z=0}^{1} F(z, s) \sin ^{2}(\pi z) d z$ and $R_{2}^{*}=\frac{R_{2}}{R_{0 c}}$.

We now move on to consider the effect of gravity modulation on magneto-convection in the absence of temperature modulation.

\section{Gravity modulation}

When the physical configuration of the magneto-convection problem is vibrated time-periodically in the $z$-direction then the 
effective gravity takes the form:

$\vec{g}(t)=g_{0}\left[1+\varepsilon^{2} \delta_{1} \cos (\omega t)\right] \hat{k}$,

where $g_{0}$ is the mean gravity, $\delta_{1}$ is the small amplitude of gravity modulation, $\omega$ is the frequency and $t$ is the time.

The governing equations for the magneto-convection are same as Eqs. (1)-(5) but with $g$ given by Eq. (46). In the absence of temperature modulation, the basic state temperature distribution is given by

$T_{b}=1-z$.

Following the procedure of the previous section, the non-dimensional form of the perturbation equations, on using Eq. (46) is:

$\nabla \cdot \vec{q}=0$

$\frac{1}{\operatorname{Pr}}\left[\frac{\partial \vec{q}}{\partial t}+(\vec{q} \cdot \nabla) \vec{q}\right]-Q P m(\vec{H} \cdot \nabla) \vec{H}=-\nabla P+R a(t) T \hat{k}+\nabla^{2} \vec{q}$,

$\left(\frac{\partial}{\partial t}-\nabla^{2}\right) T+(\vec{q} \cdot \nabla) T=0$,

$\left(\frac{\partial}{\partial t}-\nabla^{2}\right) \vec{H}+(\vec{q} \cdot \nabla) \vec{H}-(\vec{H} \cdot \nabla) \vec{q}=0$,

where $R a(t)=R_{0}\left[1+\varepsilon^{2} \delta_{1} \cos (\Omega s)\right]$. The other parameters in the above equations are as defined in the nomenclature. Now applying the solvability condition as in the previous section, we get the Ginzburg-Landau equation in the form

$$
\begin{aligned}
{\left[\frac{\delta^{2}}{\operatorname{Pr}}+\frac{k_{c}^{2}}{\delta^{4}} R_{0}-\frac{Q \pi^{2}}{P m \delta^{2}}\right]\left(\frac{d A}{d s}\right)=} & \frac{k_{c}^{2}}{\delta^{2}} R_{0}\left[\delta_{1} \cos (\Omega s)+R_{2}^{*}\right] A(s) \\
& -\left[\frac{k_{c}^{4}}{8 \delta^{4}}-\frac{Q \pi^{4}}{2 P m^{2} \delta^{2}}\left(\frac{1}{2}-\frac{k_{c}^{2}}{\delta^{2}}\right)\right] A^{3} .
\end{aligned}
$$

Eq. (52) has been solved numerically as in the previous section.

\section{Results and discussion}

In this paper we consider the individual effect of temperature and gravity modulations on heat transport by stationary magnetoconvection in Newtonian liquids. The effects of temperature and gravity modulations are represented respectively by amplitudes $\delta_{2}$ and $\delta_{1}$. The effects of electrical conductivity and magnetic field come through $P m$ and $Q$. There is the property of the fluid coming into the picture as well through the Prandtl number Pr.

We concentrate on the modulated problem for only the direct mode. The focus in the paper is essentially on the effect of modulation on heat transport. In both the modulated problems considered, the Ginzburg-Landau equation is non-autonomous. We first discuss the results on temperature modulation and then on gravity modulation.

Three types of temperature modulation are considered:

1. In-phase modulation (IPM) $(\phi=0)$.

2. Out-of-phase modulation (OPM) $(\phi=\pi)$.

3. Modulation only of the lower boundary (MOLB) $(\phi=-i \infty)$.

From linear theory of the problem that can be taken from Siddheshwar and Pranesh [36], the following results are available.

1. $R_{0 c}$ is same for all values of $P r$ and $P m$ as we consider only the stationary mode of convection that is possible in conducting liquids in which $\mathrm{Pr}<\mathrm{Pm}$.

2. $R_{0 c}(Q=0)<R_{0 c}(Q \neq 0)$.
3. (a) $R_{0 c}\left(\delta_{2}=0\right)<R_{0 c}\left(\delta_{2} \neq 0\right)$ for IPM (in phase modulation $\phi=0)$.

(b) $R_{0 c}\left(\delta_{2}=0\right)>R_{0 c}\left(\delta_{2} \neq 0\right.$ ) for OPM (out phases modulation $\phi=\pi)$.

(c) $R_{0 c}\left(\delta_{2}=0\right)>R_{0 c}\left(\delta_{2} \neq 0\right.$ ) for MOLB (modulation at lower plate $\phi=-i \infty)$.

4. $R_{0 c}\left(\delta_{1}=0\right)<R_{0 c}\left(\delta_{1} \neq 0\right)$.

A weakly non-linear study pursued in the paper using Ginzburg-Landau equation reveals many new results on heat transport, which have been presented in terms of the Nusselt number $\mathrm{Nu}(\mathrm{s})$. The effect of each one of these modulations on heat transfer, are shown in Figs. 2-5.

a

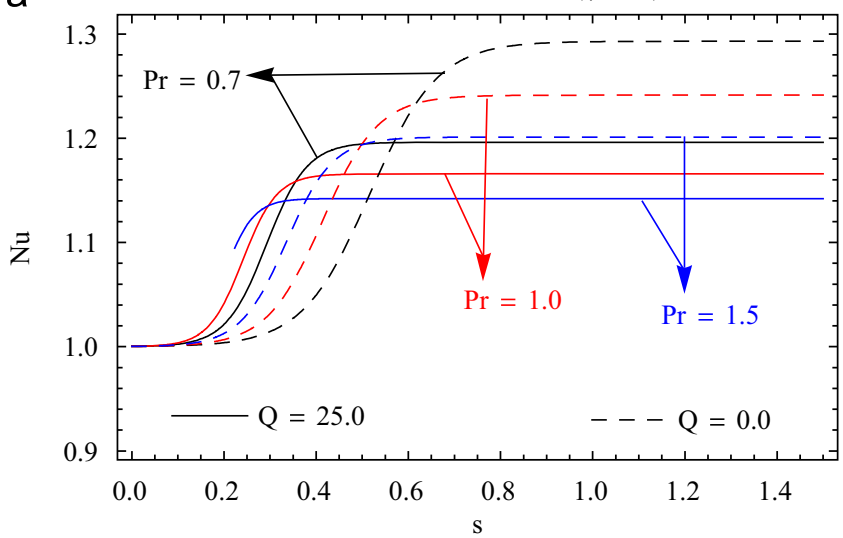

b

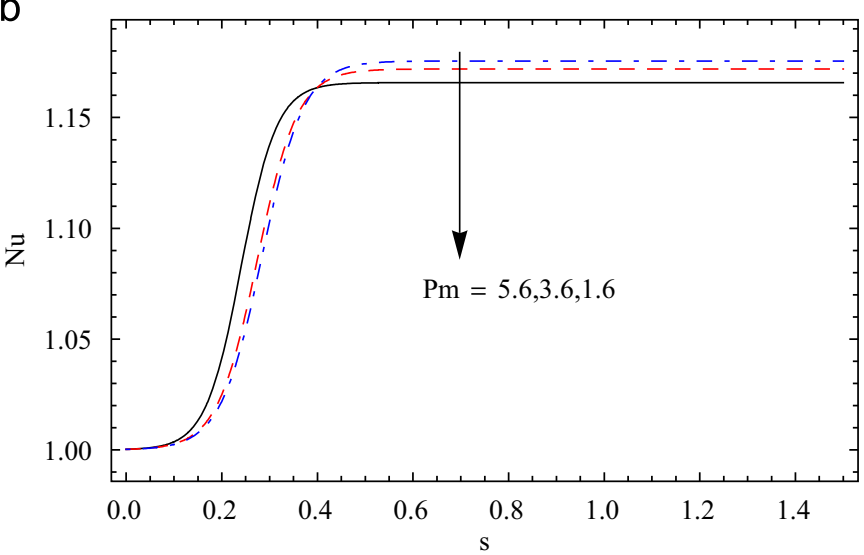

C

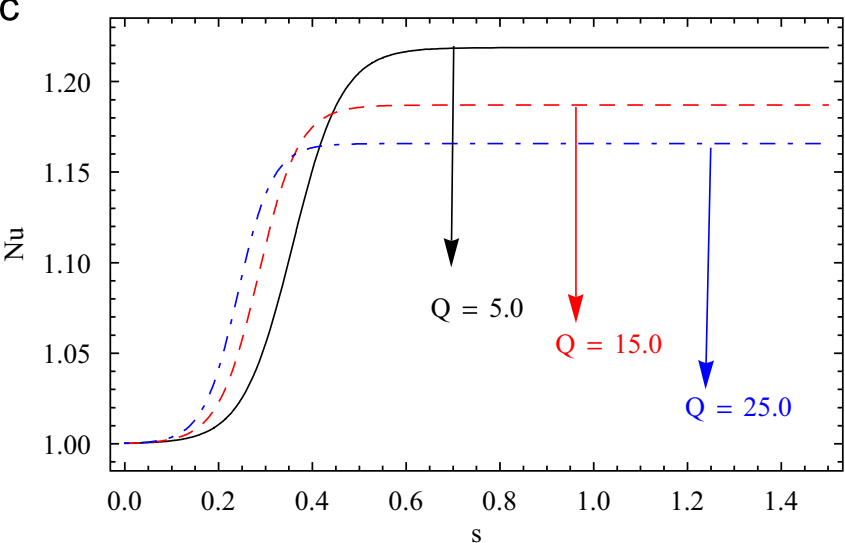

Fig. 2. $N u$ versus time (s). (a) $P r, P m=1.6, \delta_{2}=0.05, \omega=2$. (b) $P m, P r=1.0$ $P m=1.6, \delta_{2}=0.05, \omega=2$. (c) $Q, Q=25.0, P r=1.0, \delta_{2}=0.05, \omega=2$. 

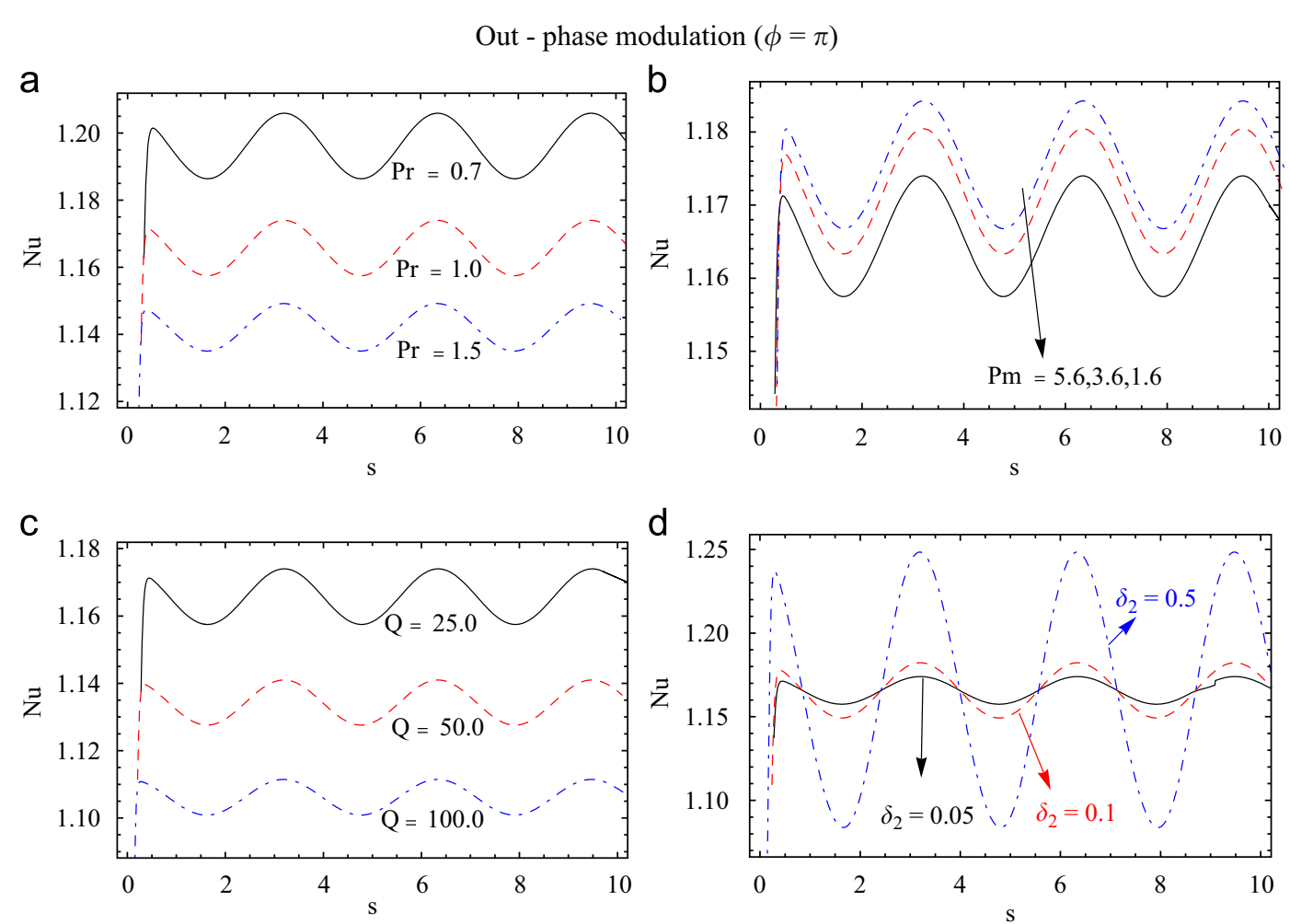

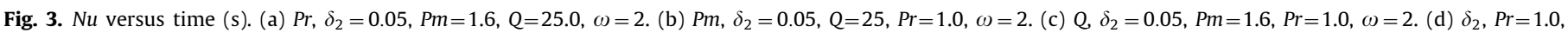
$P m=1.6, Q=25.0, \omega=2$.
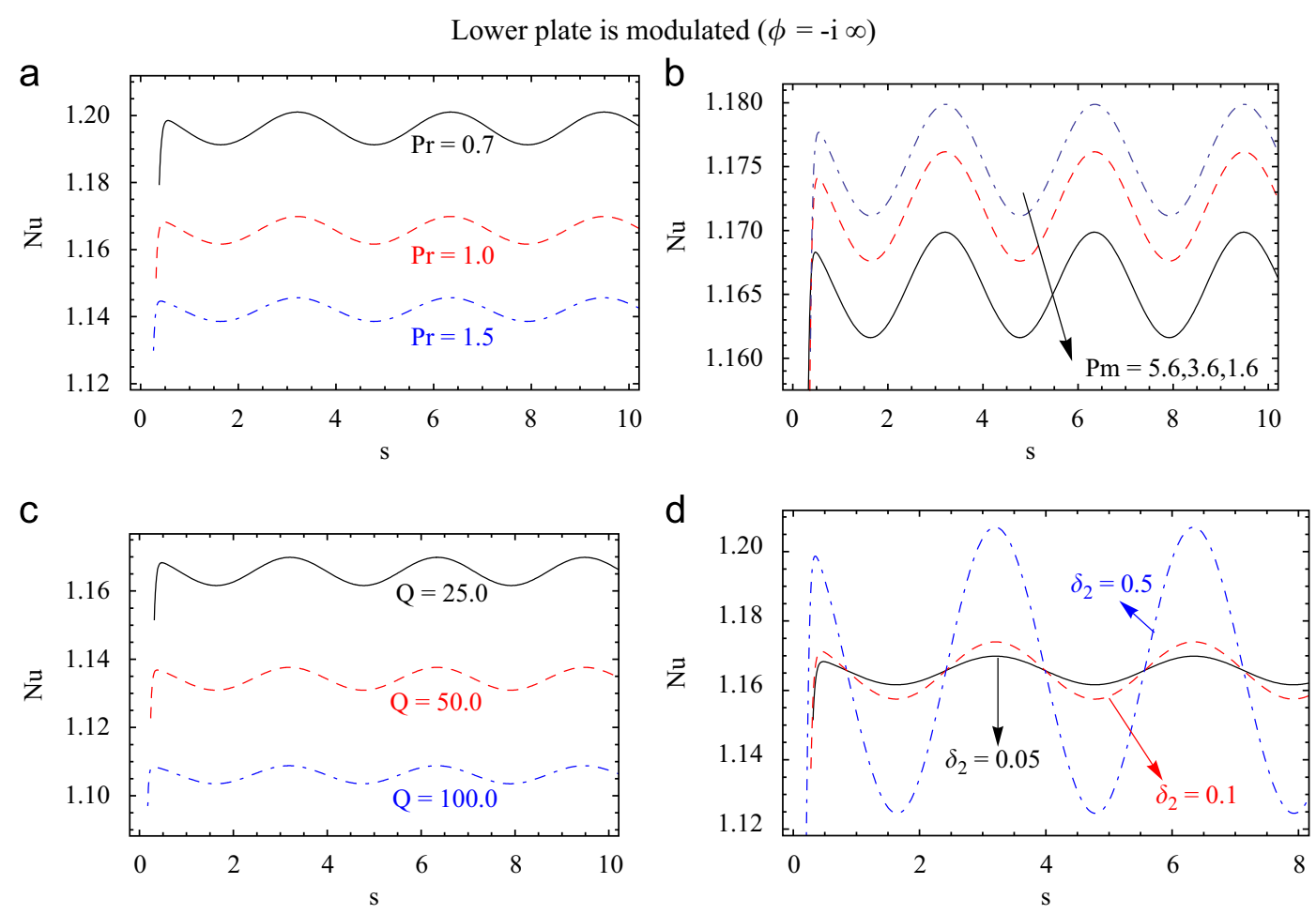

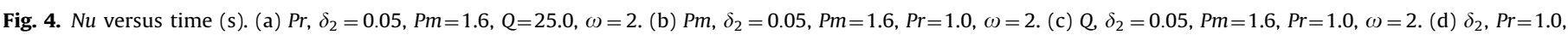
$P m=1.6, Q=25.0, \omega=2$.

Fig. 2(a)-(c) concerning IPM shows that initially when $t=0$ the value of $\mathrm{Nu}$ is 1.0, which shows the conduction state of the system. As the value of $t$ increases the value of $N u$ also increases. Then the $\mathrm{Nu}$ versus $\tau$ curves level off after some time. This result is seen when the amplitude of temperature modulation is quite small. The above pattern of variation is also seen in the case of OPM (see Fig. 3(a)-(e)) and MOLB (see Fig. 4(a)-(e)). In the OPM and MOLB cases, however, the $\mathrm{Nu}$ versus $\tau$ curves are oscillatory. 
Gravity Modulation

a

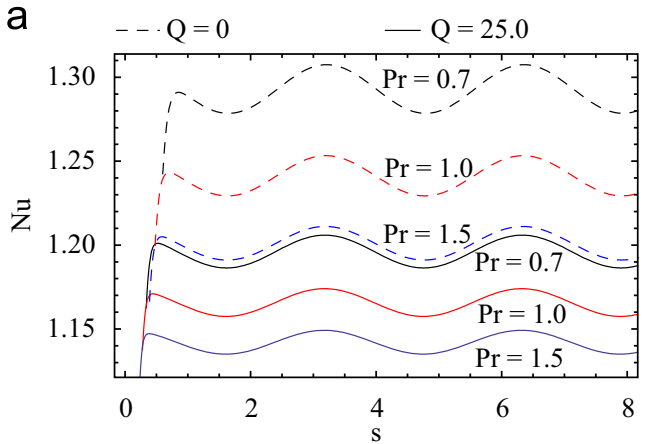

C

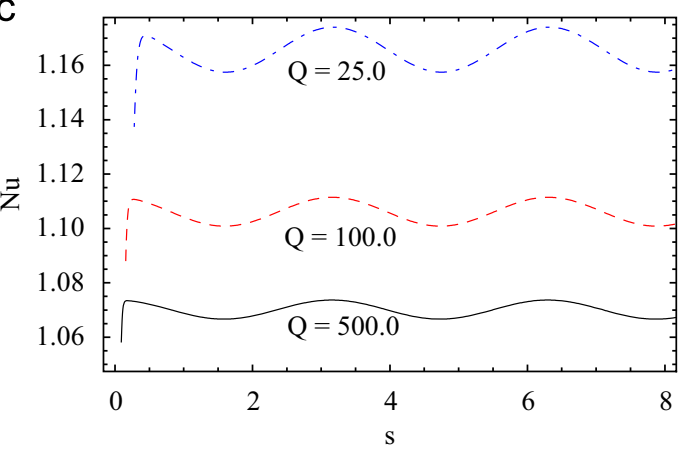

b

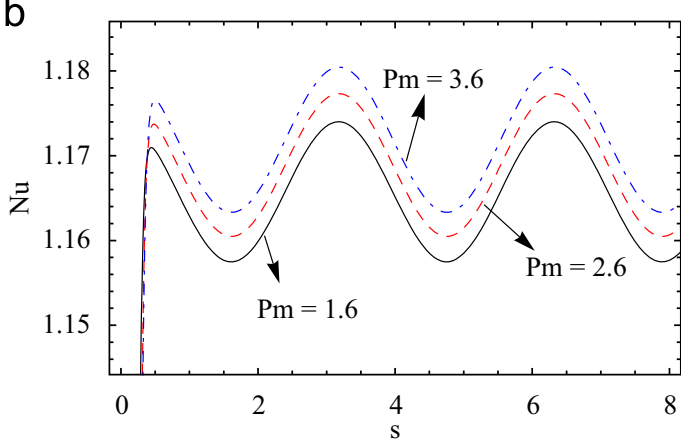

d

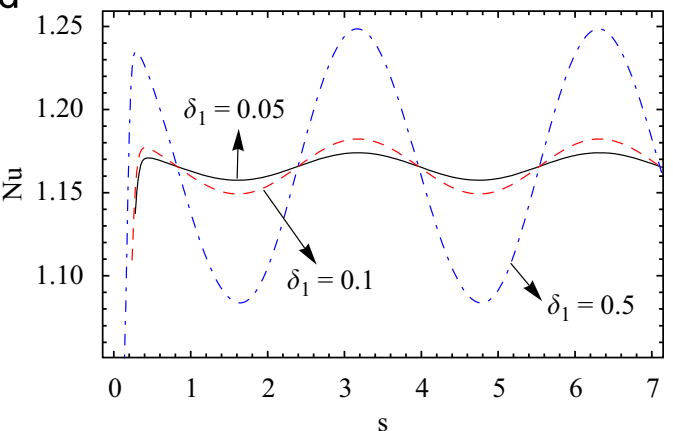

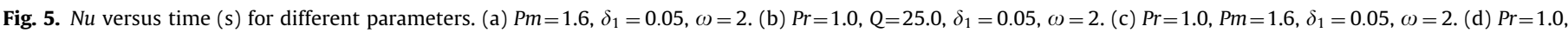
$Q=25.0, P m=1.6, \omega=2$.

From these figures the following general result is also apparent: $N u^{I P M}<N u^{M O L B}<N u^{O P M}$

Fig. 3(d) shows the effect of amplitude of temperature modulation on $\mathrm{Nu}$ in the case of OPM. It is obvious from the figure that

$\mathrm{Nu} / \delta_{\delta_{2}=0.05}<\mathrm{Nu} / \delta_{\delta_{2}=0.1}<\mathrm{Nu} / \delta_{\delta_{2}=0.5}$.

Fig. 4(d) reveals that this result is true in the case of MOLB also.

Further we have some more results on heat transport quantified in the form of Nusselt number $\mathrm{Nu}(\mathrm{s})$ :

1. $N u(P r=0.7)>N u(P r=1.0)>N u(P r=1.5)$ (see Figs. 2(a), 3(a), $4(\mathrm{a})$, and $5(\mathrm{a}))$.

2. $N u(P m=1.6)<N u(P m=2.6)<N u(P m=3.6)$ (see Figs. 2(b), 3(b), 4(b), and 5(b)).

3. $N u(Q=0)>N u(Q \neq 0)$ (see Figs. 2(c), 3(c), 4(c), and 5(c)).

4. (a) $N u\left(\delta_{2}=0\right)<N u\left(\delta_{2} \neq 0\right)$ for OPM (see Fig. 3(d)).

(b) $N u\left(\delta_{2}=0\right)<N u\left(\delta_{2} \neq 0\right)$ for MOLB (see Fig. 4(d)).

5. $N u\left(\delta_{1}=0\right)<N u\left(\delta_{1} \neq 0\right)$ for gravity modulation (see Fig. $\left.5(\mathrm{~d})\right)$.

\section{Conclusions}

The effect of temperature/gravity modulation on the onset of magneto-convection in a viscous fluid is studied using GinzburgLandau equation. An onset criterion for weak non-linear convection is derived analytically. The following conclusions are drawn:

1. Effect of increasing $Q$ and/or $P r$ is to decrease $N u$ and thus the heat transport.

2. On increasing the value of $P m$, or $\delta_{1}$, or $\delta_{2}$ convection increases and hence amount of heat transport.

3. It is clear from the graphs that magnetic field stabilizes the system.
4. The value of $N u$ in case of MOLB is less than that of OPM but greater than that of IPM.

5. The results of IPM are non-oscillatory. However in cases of OPM and MOLB they are oscillatory and similar to that of gravity modulation.

\section{Acknowledgment}

Part of this work was done during the lien period sanctioned to the author B.S.B. by the Banaras Hindu University, Varanasi, India to work as Professor of Mathematics at Department of Applied Mathematics and Statistics, Babasaheb Bhimrao Ambedkar University, Lucknow, India. Pankaj Mishra is highly thankful to Council of Scientific and Industrial Research, New Delhi for providing financial support to carry out the present research work.

\section{References}

[1] S.H. Davis, The stability of time periodic flows, Annual Review of Fluid Mechanics 8 (1976) 57-74.

[2] G. Venezian, Effect of modulation on the onset of thermal convection, Journal of Fluid Mechanics 35 (1969) 243-254.

[3] S. Rosenblat, G.A. Tanaka, Modulation of thermal convection instability, Physics of Fluids 14 (1971) 1319-1322.

[4] M.H. Roppo, S.H. Davis, S. Rosenblat, Bénard convection with time periodic heating, Physics of Fluids 27 (1984) 796-803.

[5] B.S. Bhadauria, P.K. Bhatia, Time-periodic heating of Rayleigh-Benard convection, Physica Scripta 66 (1) (2002) 59-65.

[6] B.S. Bhadauria, Time-periodic heating of Rayleigh-Benard convection in a vertical magnetic field, Physica Scripta 73 (3) (2006) 296-302.

[7] B.S. Bhadauria, P.K. Bhatia, L. Debnath, Weakly non-linear analysis of Rayleigh-Benard convection with time periodic heating, International Journal of Non-Linear Mechanics 44 (2009) 58-65.

[8] P.M. Gresho, R.L. Sani, The effects of gravity modulation on the stability of a heated fluid layer, Journal of Fluid Mechanics 40 (1970) 783-806. 
[9] G.Z. Gershuni, E.M. Zhukhovitskii, I.S. Iurkov, On convective stability in the presence of periodically varying parameter, Journal of Mechanics and Applied Mathematics 34 (1970) 470-480.

[10] S. Biringen, L.J. Peltier, Numerical simulation of 3D Bénard convection with gravitational modulation, Physics of Fluids (A) 2 (1990) 754-764.

[11] R. Clever, G. Schubert, F.H. Busse, Two-dimensional oscillatory convection in a gravitationally modulated fluid layer, Journal of Fluid Mechanics 253 (1993) 663-680.

[12] Y. Shu, B.Q. Li, B.R. Ramaprian, Convection in modulated thermal gradients and gravity: experimental measurements and numerical simulations, International Journal of Heat Mass Transfer 48 (2005) 145-160.

[13] R. Clever, G. Schubert, F.H. Busse, Three-dimensional oscillatory convection in a gravitationally modulated fluid layer, Physics of Fluids (A) 5 (1993) 2430-2437.

[14] J.L. Rogers, W. Pesch, O. Brausch, M.F. Schatz, Complex-ordered patterns in shaken convection, Physical Review (E) 71 (2005) 066214 (1-18).

[15] S. Aniss, J.P. Brancher, M. Souhar, Asymptotic study and weakly non-linear analysis at the onset of Rayleigh-Bénard convection in Hele-Shaw cell, Physics of Fluids 7 (5) (1995) 926-934.
[16] S. Aniss, M. Souhar, M. Belhaq, Asymptotic study of the convective parametric instability in Hele-Shaw cell, Physics of Fluids 12 (2) (2000) 262-268.

[17] B.S. Bhadauria, P.K. Bhatia, L. Debnath, Convection in Hele-Shaw cell with parametric excitation, International Journal of Non-Linear Mechanics 40 (2005) 475-484.

[18] T. Boulal, S. Aniss, M. Belhaq, Effect of quasiperiodic gravitational modulation on the stability of a heated fluid layer, Physical Review E 76 (2007) 056320 (1-5).

[19] H. Ozoe, M. Maruo, Magnetic and gravitational natural convection of melted silicon. Two-dimensional numerical computations for the rate of heat transfer, JSME International Journal 30 (1987) 774-784.

[20] P.G. Siddheshwar, S. Pranesh, Effect of temperature/gravity modulation on the onset of magneto-convection in electrically conducting fluids with internal angular momentum, Journal of Magnetism Magnetic Materials 219 (2000) 153-162. 